\title{
State of EB Accelerator Technologies \& Future Opportunities
}

Charles Thangaraj and Gianluigi Ciovati 


\section{Existing industrial accelerators}




\section{Accelerators comes in several sizes and shapes.}

- Electrostatic (few keV - $10 \mathrm{MeV}$ ) - e.g. Dyanmitron, Cockroft-Walton, Pelletron

- Microtron - a cross of cyclotron but uses multi-pass

- Betatron - essentially a transformer but circular can reach several MeV's

- Rhodotron - recirculating through a coaxial cavity

- RF Linac (several MeV's) - normal conducting cavities

- Synchrotron

- Ion accelerators (different species)

A steady market 


\section{Commercial EB accelerator applications are vast}

- EB welding

- EB melting

- EB sterilization

- EB curing

- Non-destructive testing

- Medical imaging

- Cargo inspection 


\section{New technology: Compact SRF accelerator concepts}




\section{Current vs New Accelerator Technology}

- Bulk materials processing applications require multi-Mev energy for penetration and 100 's of kW (or even MW) of beam power

- > few MeV accelerators are typically copper and RF driven

- Inherent losses limit efficiency (heat vs beam power) = ops cost

- Heat removal limits duty factor, gradient and average power $\rightarrow$ physically large "fixed" installations $=$ CAPEX

\section{New Technology: Superconducting Radio Frequency (SRF)}

- High wall plug power efficiency (e.g. 75\%)

- Large fraction of the input power goes into beam

- High power \& efficiency enables new \$1 Billion class SRF-based science machines $\rightarrow$ driving large R\&D efforts at labs

- Currently SRF-based science accelerators are huge with complex cryogenic refrigerators, cryomodules, etc. But this is changing!
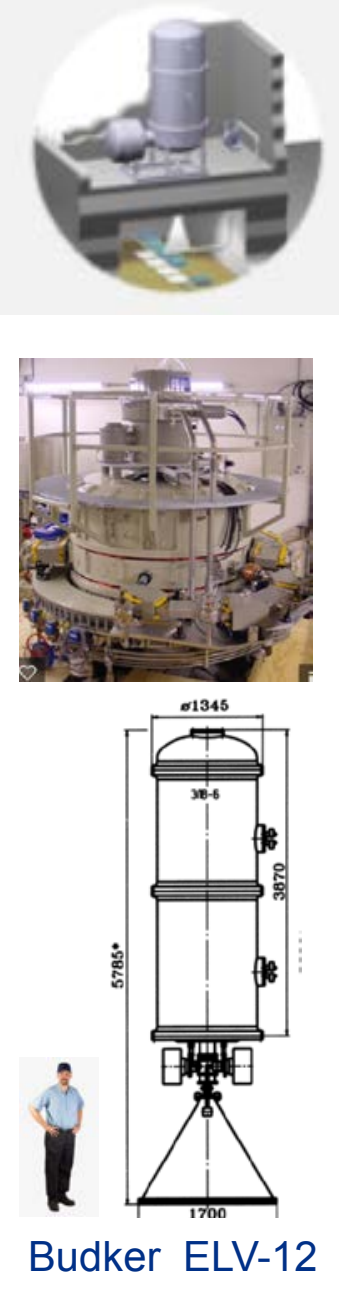

- Recent SRF breakthroughs now enable a new class of compact, SRF-based industrial accelerators (lower CAPEX and OPS cost) 


\section{Superconducting radio-frequency accelerator technology}

- Superconducting radio-frequency cavities are building blocks of modern particle accelerators

- Much higher efficiency in converting RF power into beam power than copper cavities

- Standard technology: bulk $\mathrm{Nb}$, cooled at $2-4 \mathrm{~K}$

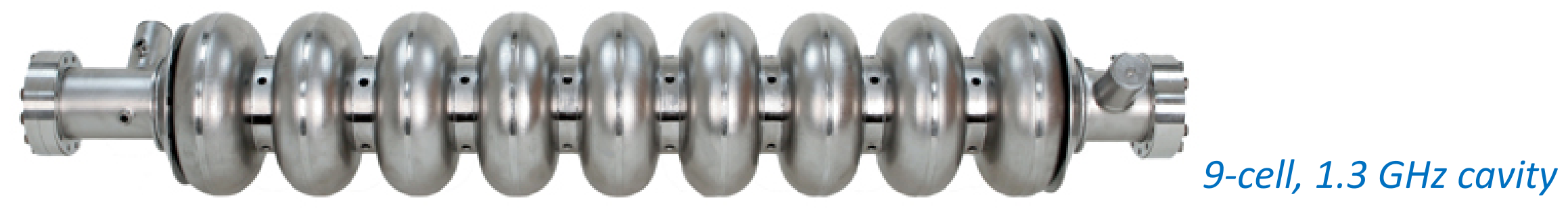

- Recent advances in SRF R\&D make possible the use of $\mathrm{Nb}_{3} \mathrm{Sn}$ thin film operating at $\geq 4 \mathrm{~K}$ with higher efficiency than that of bulk $\mathrm{Nb}$ 


\section{Design commonalities}

- Thermionic gun for high-current beam

- Cryostat with $\mathrm{Nb}_{3} \mathrm{Sn}$ SRF cavity for efficient acceleration

- Cryocoolers for efficient cooling

- Coaxial input power couplers for efficient coupling of RF into cavity

- Beam transport calculation and thermal analysis verified feasibility of the designs 


\section{Solicitation for advancing industrial accelerators}

- Dept. of Energy provided funding to develop novel accelerator designs to address need for industrial application in the energy and environment sectors

\begin{tabular}{|c|c|c|c|c|}
\hline & $\begin{array}{l}\text { Type } 1 \\
\text { Demo/Small } \\
\text { Scale }\end{array}$ & $\begin{array}{l}\text { Type } 2 \\
\text { Medium } \\
\text { Scale Low } \\
\text { Energy }\end{array}$ & $\begin{array}{l}\text { Type } 3 \\
\text { Medium Scale } \\
\text { High Energy }\end{array}$ & $\begin{array}{l}\text { Type } 4 \\
\text { Large Scale } \\
\text { High Energy }\end{array}$ \\
\hline $\begin{array}{l}\text { Example } \\
\text { Applications }\end{array}$ & $\begin{array}{l}\text { R\&D, } \\
\text { Sterilization, } \\
\text { industrial } \\
\text { effluent streams }\end{array}$ & $\begin{array}{l}\text { Flue Gas, } \\
\text { Waste water }\end{array}$ & $\begin{array}{l}\text { Wastewater, } \\
\text { sludge, medical } \\
\text { waste }\end{array}$ & $\begin{array}{l}\text { Sludge, Medical } \\
\text { waste, Env. } \\
\text { remediation }\end{array}$ \\
\hline $\begin{array}{l}\text { Electron Beam } \\
\text { Energy }\end{array}$ & $0.5-1.5 \mathrm{MeV}$ & $1-2 \mathrm{MeV}$ & $10 \mathrm{MeV}$ & $10 \mathrm{MeV}$ \\
\hline $\begin{array}{l}\text { Electron Beam } \\
\text { Power }(\mathrm{CW})\end{array}$ & $>0.5 \mathrm{MW}$ & $>1 \mathrm{MW}$ & $>1 \mathrm{MW}$ & $>10 \mathrm{MW}$ \\
\hline $\begin{array}{l}\text { Wallplug } \\
\text { Efficiency }\end{array}$ & $>50 \%$ & $>50 \%$ & $>50 \%$ & $>75 \%$ \\
\hline $\begin{array}{l}\text { Target Capital } \\
\text { Cost* }\end{array}$ & $<\$ 10 / W$ & $<\$ 10 / W$ & $<\$ 10 / W$ & $<\$ 5 / W$ \\
\hline $\begin{array}{l}\text { Target Operating } \\
\text { Cost } \uparrow\end{array}$ & $<1.0 \mathrm{M} \$ / \mathrm{yr}$ & $<1.5 \mathrm{M} \$ / \mathrm{yr}$ & $<1.5 \mathrm{M} \$ / \mathrm{yr}$ & $<12 \mathrm{M} \$ / \mathrm{yr}$ \\
\hline
\end{tabular}


$1 \mathrm{MeV}, 1 \mathrm{MW}$ SRF accelerator

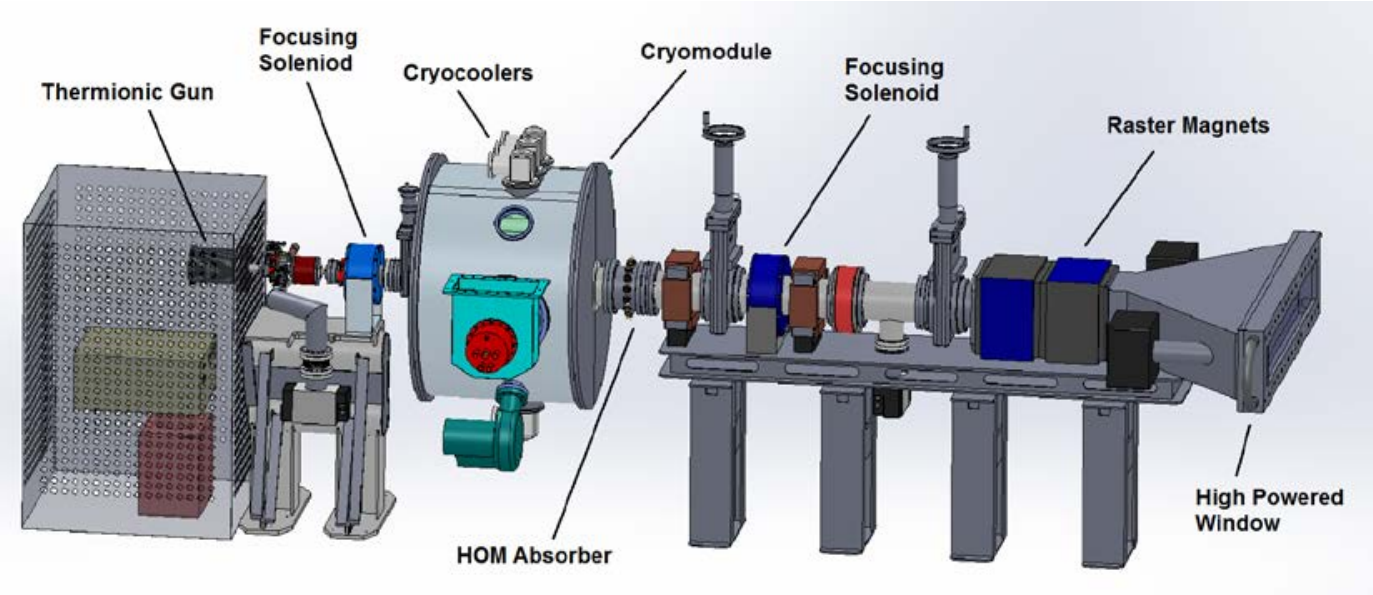

Jefferson Lab G. Ciovati, R. Rimmer, F. Hannon, J. Guo, F. Marhauser, V. Vylet

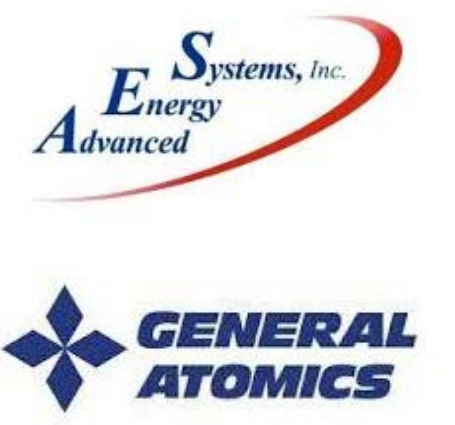

J. Rathke, T. Schultheiss

\section{$10 \mathrm{MeV}, 1 \mathrm{MW}$ SRF accelerator}

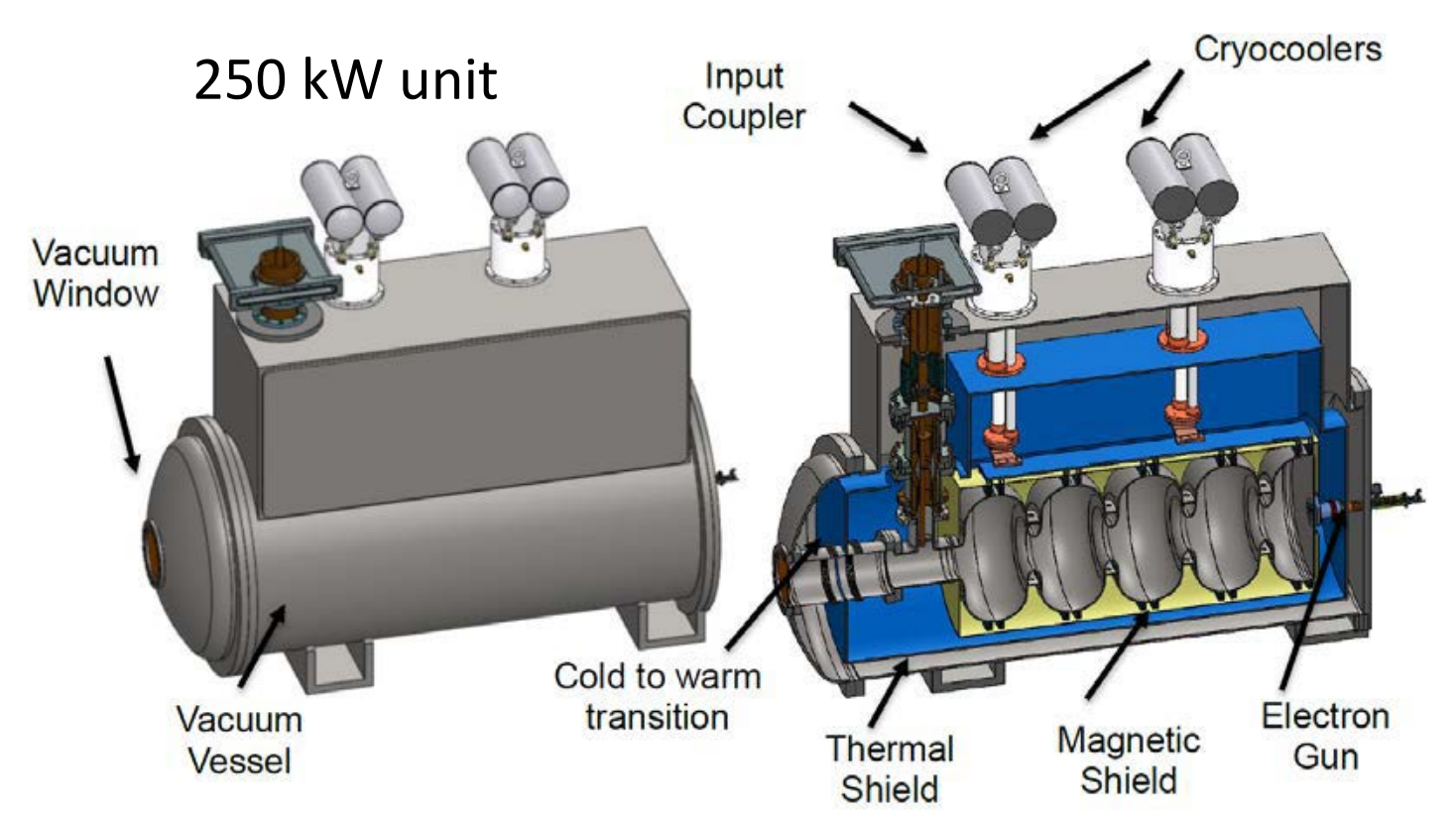

莫Fermilab 


\section{Facilities Layout}

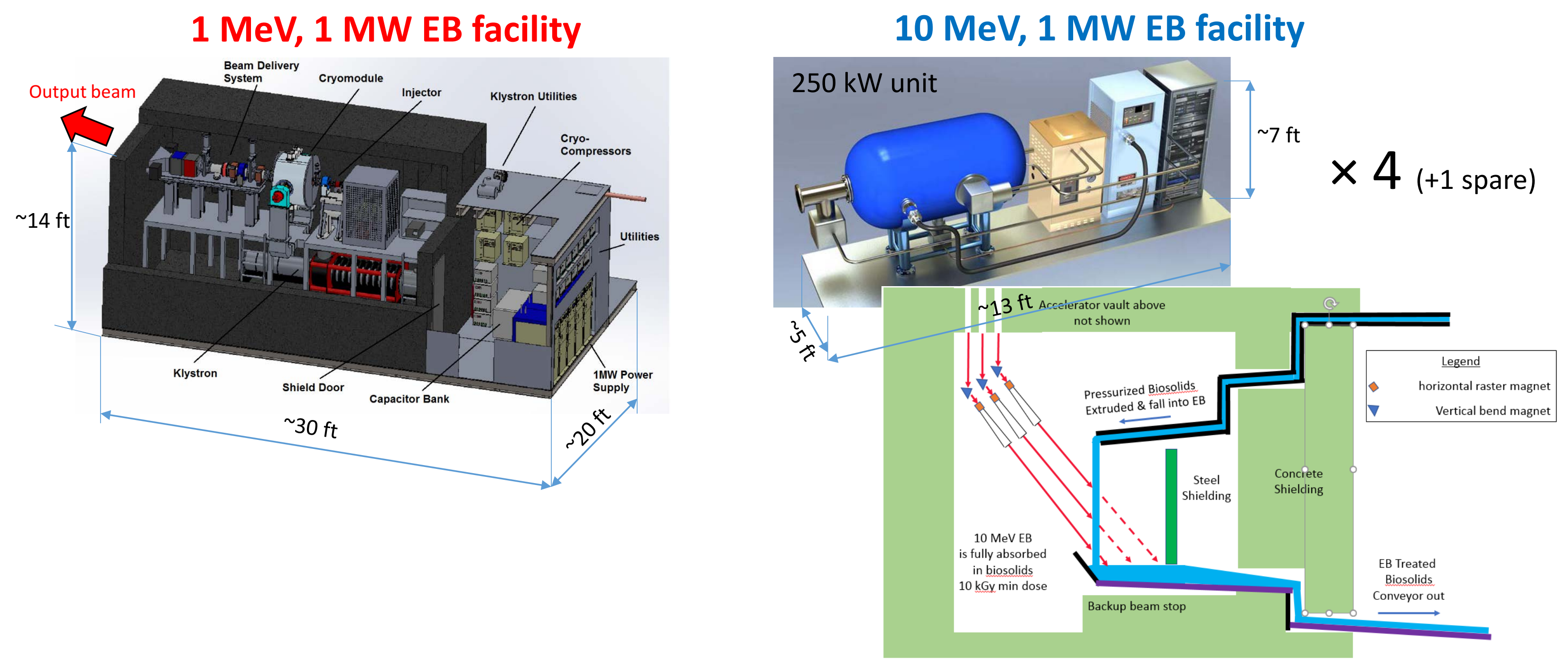




\section{New opportunities with compact industrial SRF-based accelerators}




\section{Future Accelerator Applications}

\section{Energy and Environment}

- Treat Municipal Waste \& Sludge

- Eliminate pathogens in sludge

- Destroy organics, pharmaceuticals in waste water

- In-situ environmental remediation

- Contaminated soils

- Spoils from dredging, etc

\section{Industrial and Security}

- Catalyze Chemical reactions to save time and energy

- In-situ cross-link of materials

- Improve pavement lifetime

- Instant cure coatings

- Medical sterilization without Co60

- Improved non-invasive inspection of cargo containers

These new applications need cost effective, energy efficient, high average power electron beams.

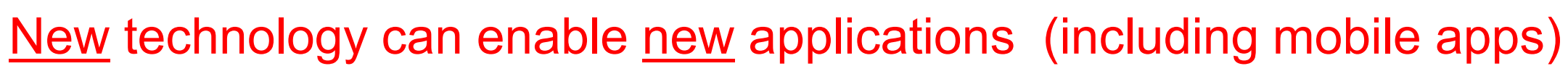




\section{Economics of SRF E-beam treatment}




\section{Cost estimate for $1 \mathrm{MeV}, 1 \mathrm{MW}$ SRF EB facility}

\begin{tabular}{|l|l|}
\hline Capital Cost & \\
\hline SRF Accelerator & $\$ 4,500,000$ \\
\hline Infrastructure & $\$ 2,750,000$ \\
\hline Total & $\$ 7,250,000$ \\
\hline Investment (20\%) & $\$ 1,450,000$ \\
\hline Amortization(15yr @ 8\%) & $\$ 670 \mathrm{k} / \mathrm{yr}$ \\
\hline
\end{tabular}

\begin{tabular}{|c|c|}
\hline Operating Cost $(8,000 \mathrm{hrs}$ & \\
\hline Powera) & $\$ 159.2 / \mathrm{hr}$ \\
\hline Cooling water & None (air-cooled chillers) \\
\hline Maintenance $^{b)}$ & $\$ 145 \mathrm{k} / \mathrm{yr}$ \\
\hline Total & $\$ 1,418,600 / y r$ \\
\hline Total Cost (Capital + Op.) & $\begin{array}{l}\$ 261 / \mathrm{hr} \\
\$ 2,088,600 / \mathrm{yr}\end{array}$ \\
\hline
\end{tabular}

\section{Assumptions}

a) $2.274 \mathrm{MW}$ (Elec. Eff.: 42\%) @ \$0.07/kWh

b) $2 \%$ capital/year

c) No dedicated operator

Electric powe

Amortization

Maintenance

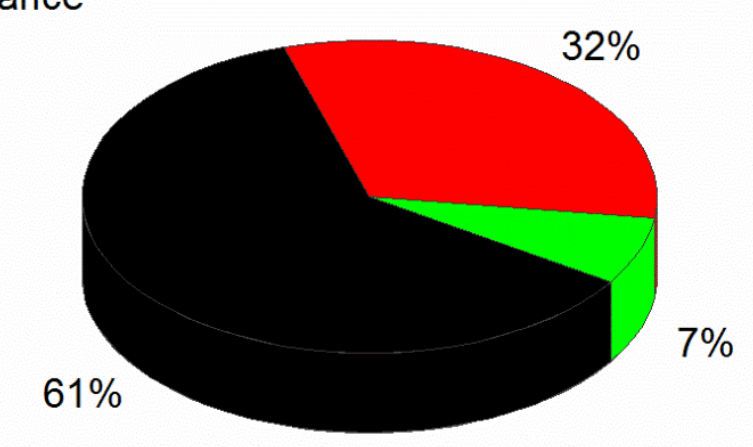




\section{Processing cost sensitivity to Design Parameters}
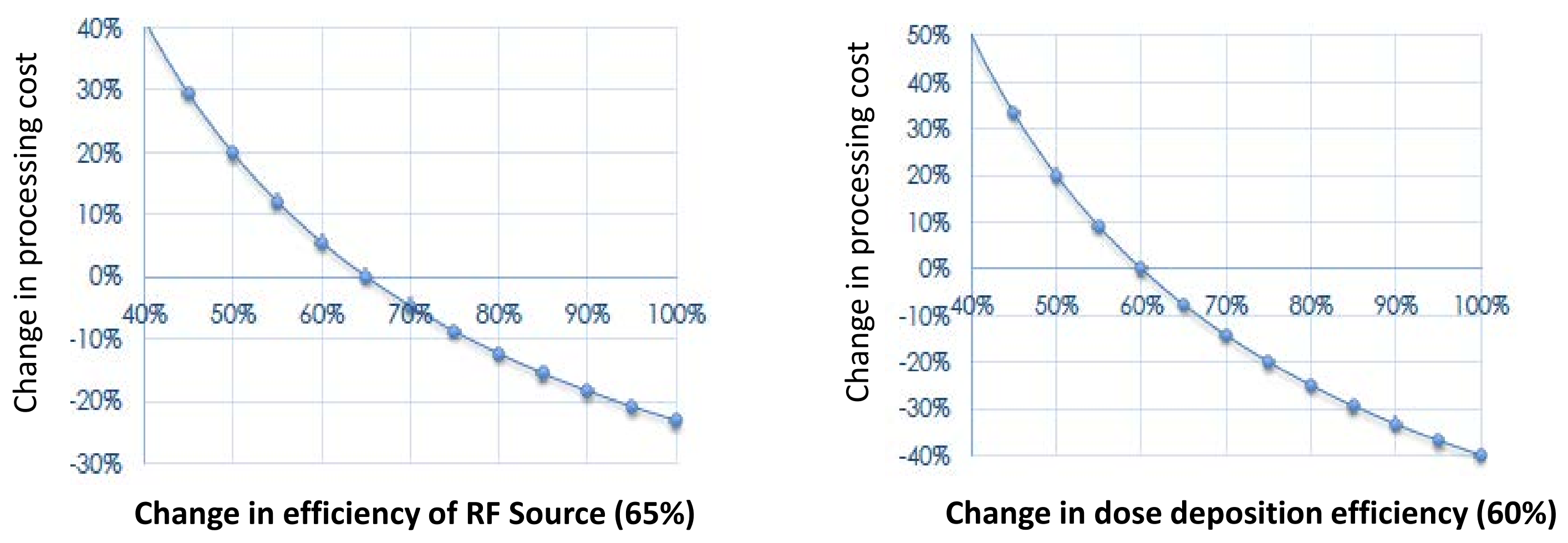

Current technology: klystron (65\%), IOT (70\%)

In development: magnetrons (90\%) 


\section{Processing cost sensitivity to Operation Parameters}
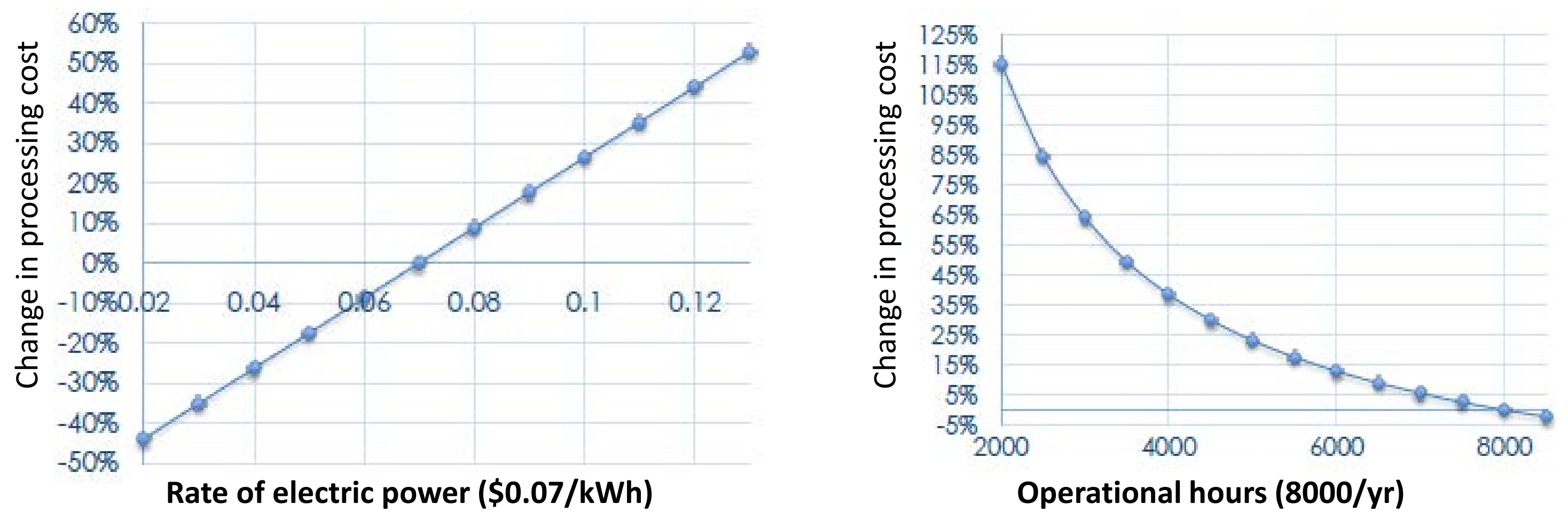


\section{Processing cost per Application}

\begin{tabular}{|l|c|c|c|} 
& \multicolumn{2}{|c|}{$1 \mathrm{MeV}, 1 \mathrm{MW}$} & $10 \mathrm{MeV}, 1 \mathrm{MW}$ \\
\hline & \multicolumn{2}{|c|}{ WASTEWATER } & SLUDGE \\
\hline Dose requirement & $1 \mathrm{kGy}$ & $4 \mathrm{kGy}$ & $10 \mathrm{kGy}$ \\
\hline \begin{tabular}{l|l} 
Processing cost \\
Cost of current technologies \\
(other than EB) $[4]$
\end{tabular} & $\$ 0.13 / \mathrm{m}^{3}(\$ 0.482 / \mathrm{kgal})$ & $\$ 0.51 / \mathrm{m}^{3}(\$ 1.93 / \mathrm{kgal})$ & $\$ 19.7 / \mathrm{dry}$ ton \\
\hline
\end{tabular}

\begin{tabular}{|l|c|c|c|}
\hline Daily Processed Volume & $\begin{array}{c}45,000 \mathrm{~m}^{3} \\
(11.9 \mathrm{Mgal})\end{array}$ & $\begin{array}{c}11,250 \mathrm{~m}^{3} \\
(3.0 \mathrm{Mgal})\end{array}$ & $\begin{array}{c}278 \text { dry ton (1.3 Mgal } \\
\text { with 25\% biosolid } \\
\text { waste) }\end{array}$ \\
\hline Required Flow Rate (gpm) & 9,050 & 2,260 & 984 \\
\hline Comments [4] & $\begin{array}{c}\text { Color, Odor, Coliform } \\
\text { bacteria removal }\end{array}$ & Kill $>99 \%$ of bacteria & $\begin{array}{c}\text { Inactivate some } \\
\text { radiation resistant } \\
\text { organisms }\end{array}$ \\
\hline
\end{tabular}

